\title{
ESTUDO DO SALTO VERTICAL MÁXIMO: ANÁLISE DA CORRELAÇÃO DE FORÇAS APLICADAS
}

Emerson Miguel da Cruz;

Antonia Dalla Pria Bankoff

\section{Resumo}

As variáveis estudadas foram: a altura máxima atingida, a potência, a força de saída do solo, a força de chegada no solo, a força relativa e a velocidade. Foi usado um sistema de plataforma de força com câmaras de vídeo e infravermelho sincronizadas a este o que permitiam a obtenção dos valores de força e a reprodução virtual dos saltos. Participaram do estudo 15 sujeitos do sexo masculino com idades entre 17 e 35 anos, atletas de handebol da cidade de Goiânia. Cada sujeito realizou três saltos verticais máximos estilo "counter moviment jump". A análise correlacional mostrou que a variável que mais se relacionou com a máxima impulsão vertical foi a velocidade $(\mathrm{r}=0,99 ; \mathrm{P}<0,001$ e $\mathrm{r} 2=99 \%)$ e a variável que mostrou maior relação com a potência foi a força de saída do solo $\left(\mathrm{r}=0,85 ; \mathrm{P}<0,001\right.$ e $\left.\mathrm{r}^{2}=73,28\right)$. A análise comparativa entre os maiores e os menores resultados para o salto nos mostra que o primeiro grupo conseguiu resultados $37,6 \%$ maior que o segundo grupo e as variáveis que mais contribuíram para isto foram a velocidade e a potência.

\section{Palavras-Chave}

Salto vertical; Força; Velocidade; Potência; Correlação.

\section{STUDY OF VERTICAL JUMP MAXIMUM: ANALYSES RELATIONSHIP}

Emerson Miguel da Cruz;

Antonia Dalla Pria Bankoff

\begin{abstract}
The researched variations were: the maximum height reached, the power, the ground reaction forces applied in the moment the subject touches and leaves the ground and the velocity. It was used a force platform system with video cameras and infrared synchronized to it, allowing the achievement of the forces values and the virtual reproduction of the jumps. The subjects for the study were 15 male handball athletes from the city of Goiânia between 17 and 35 years old. Each subject performed three maximum countermovement jump. The velocity was the variety with the closest relationship with the maximum

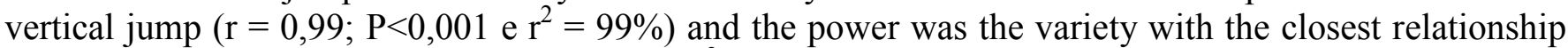
with the take off force $\left(\mathrm{r}=0,85 ; \mathrm{P}<0,001 \mathrm{e}^{2}=73,28\right)$. The comparative analyses of the biggest and lower results demonstrates that the first group had results $37.6 \%$ better than the second group, the varieties with the greater influence over it were velocity and power.
\end{abstract}

\section{Key-Words}

Vertical jump; Force; Velocity; Power; Relationship. 


\section{INTRODUÇÃO}

Segundo Durward(1) para um salto vertical e uma seqüência de aterrissagem, o sujeito geralmente começaria na posição vertical ereta e a atividade de saltar seria iniciada pela flexão do quadril e do joelho. Quanto mais baixo estiver o centro de massa, mais distância estará disponível para a fase de propulsão. Conforme o corpo é acelerado para cima, os quadris e joelhos estendem com a produção de forças no solo. Quando os membros estão completamente estendidos (incluindo a flexão plantar da articulação do tornozelo), o corpo se levanta e a fase de vôo começa. Dependendo da quantidade de trabalho realizada pelos músculos, o corpo perderá contato com o solo com certa velocidade de decolagem. Esta velocidade de decolagem será reduzida pela ação da aceleração gravitacional $\left(9,81 \mathrm{~m} / \mathrm{s}^{-}\right.$ ${ }^{2}$ ), e uma altura máxima será atingida, após isto o corpo retornará ao solo e a fase de aterrissagem será iniciada.

Dentro do ato de saltar estão envolvidas capacidades físicas importantes como: a produção de força e velocidade, que se caracterizam pela superação o mais rápido possível da resistência. A força "explosiva" representa o caso particular de manifestação das capacidades de velocidade e de força relacionadas com esforços únicos (saltos, lançamentos).(2) Formular com precisão uma definição de "força", que compreenda ao mesmo tempo seus aspectos físicos e psíquicos, ao contrário da definição dos físicos apresenta consideráveis dificuldades, pois as modalidades da força, do trabalho muscular, da contração muscular, são extremamente complexas e dependem de uma multiplicidade de fatores. ${ }^{(3)}$

A força explosiva nas condições da atividade desportiva se manifesta através de trabalho muscular isométrico e dinâmico, e no último caso, deverá ser superada a resistência externa. Sendo assim, o desportista, durante os esforços explosivos, sempre realiza ao máximo a força inicial, e o caráter da força aceleradora dependerá do valor da resistência externa e da força máxima muscular. (4)

A física nos traz muitos elementos para explicarmos os fenômenos que nos circundam, e um de seus grandes estudiosos, Isaac Newton enunciou a seguinte lei: "A variação do movimento é proporcional à força motriz e atua na direção segundo a qual a força é dirigida" Keller et al (6), o que se traduz na equação da segunda lei de Newton: somatória de força é igual à massa multiplicada pela aceleração: $\mathrm{F}=\mathrm{m} . \mathrm{a}$. 
$\overline{\text { A equação afirma que a aceleração de um objeto é proporcional à força resultante exercida sobre ele, e } \mathrm{a}}$ massa do objeto é o fator de proporcionalidade entre força resultante e a aceleração. (6)

A potência pode ser determinada como o produto da força e da velocidade, como resultado, os fatores que afetam tanto a força muscular quanto à velocidade de encurtamento determinarão à potência que pode ser produzida, fornecendo ao músculo um impulso neural adequado. Os principais determinantes da produção de potência são o número de fibras musculares ativadas em paralelo e a velocidade com que os miofilamentos podem converter energia em trabalho mecânico. A força que o músculo pode exercer é proporcional ao número de unidades geradoras de força em paralelo e a força muscular aumenta com a área de secção transversa. A velocidade máxima que um músculo pode encurtar-se é determinada pela enzima miosina ATPase. Essa enzima controla a velocidade de interação entre actina e miosina e, assim, a velocidade de ciclagem das pontes transversas. (6)

Bompa (7) afirma que a força relativa (FR) representa a razão entre a força absoluta de um atleta e seu peso corporal. Como menciona Moreira( $(8)$, a força relativa assume enorme importância quando a pessoa deve mobilizar seu corpo no espaço, baseados nesta prerrogativa e nos conceitos até aqui apresentados acredita-se que exista uma relação "ideal” entre as variáveis: potência máxima gerada, forças máximas de reação do solo, força relativa e que isto possibilite uma maior capacidade de salto vertical.

A força como uma caracterização mecânica é determinada pela direção, magnitude ou o ponto de aplicação. De acordo com a segunda lei da inércia de Newton, a força é igual à massa (m) multiplicada pela velocidade de aceleração (a), como a seguir: $\mathrm{F}=\mathrm{m} \mathrm{x}$ a. Conseqüentemente, um atleta pode melhorar a força alterando um dos dois fatores, massa ou aceleração.(7)

A seguir apresentaremos autores que estudaram o salto vertical sob os mais variados aspectos. Bosco e Komi (10); Bosco et al (11); Hudson (12); Bobbert et al (13); Pereira (14); Harman et al (15); Davies e Jones (16); Holcomb e al (17); Cordova e Armstrong (18); Landesberg e Sidemam (19); Galdi (20); Kubo et al (21); Kurokawa et al (22). Estes estudaram a determinação da composição das fibras musculares (fibras rápidas FT, e fibras lentas, ST); o ciclo de estiramento-encurtamento; o efeito combinado da energia elástica; potencial mioelétrico durante o ciclo estiramento-encurtamento; armazenamento e resposta da energia elástica em fibras lentas e rápidas dos músculos esqueléticos 
humanos e outros, contribuindo com o avanço da ciência e da tecnologia voltados para o esporte $\mathrm{e}$ qualidade de vida do homem.

\section{METODOLOGIA}

\section{População estudada}

A população estudada foi composta de quinze sujeitos do sexo masculino jogadores de handebol da cidade de Goiânia, das equipes da APCE (Associação de Pesquisa e Ciências do Esporte), do CPMG (Colégio da Polícia Militar de Goiânia) e da Associação Recreativa de Handebol de Goiânia e vários deles também são atletas da seleção universitária de handebol de Goiás; com média de idade de 25,8 anos $\pm 6,47$. Os atletas foram convidados a assinar o termo de consentimento livre e esclarecido para a pesquisa, além do preenchimento de um questionário contendo informações sobre as características gerais dos seus treinamentos, após aprovação do Comitê de Ética da Faculdade de Ciências Médicas-Unicamp, Parecer $N^{\circ} 410 / 2003$.

\section{AQUISIÇÃO DE DADOS}

Forças de reação do solo - Foi utilizado o Laboratório de Movimento da Escola Superior de Educação Física e Fisioterapia de Goiás que faz parte da Universidade do Estado de Goiás. As medidas da força de reação do solo foram registradas durante a execução de um salto vertical tipo Counter Moviment Jump (salto com contra movimento) obtidas através de um sistema de plataforma de força ligada a um computador, com seis câmeras de infravermelho sincronizadas e um sistema operacional próprio (Sistema Peak Moturs 6.0) para registro e apresentação dos valores e geração de um modelo virtual do salto executado.

Força relativa - A força relativa foi obtida: pela divisão do pico máximo de força dinâmica (reação do solo - forças de saída, obtidas na plataforma de força) pelo peso corporal do sujeito.

Impulsão Vertical - Após a realização do salto Counter Moviment Jump (salto com contra movimento), o valor da máxima altura vertical $(\mathrm{em} \mathrm{cm})$ foi obtido pelas informações do sistema da plataforma de força que nos oferece o tempo de vôo; e através da fórmula: altura é igual a um oitavo da aceleração multiplicada pelo tempo ao quadrado $\left(\mathrm{H}=1 / 8 \cdot \mathrm{a} \cdot \mathrm{t}^{2}\right)$ nos permitiu obter o valor do deslocamento vertical. 
Potência - Para o cálculo da potência média seguimos a fórmula proposta por Zatsiorsky(9) potência é igual à força multiplicada pela velocidade.

$$
\mathrm{P}=\frac{\mathrm{W}}{\mathrm{t}}=\mathrm{F} \cdot \frac{\mathrm{D}}{\mathrm{t}}=\mathrm{F} \cdot \mathrm{V}
$$

Onde $\mathrm{P}$ é igual a potência, $\mathrm{W}$ é o trabalho, $\mathrm{F}$ é a força, $\mathrm{D}$ é a distância, $\mathrm{t}$ é o tempo e $\mathrm{V}$ é a velocidade. A potência média produzida pelo sujeito durante a fase de decolagem é determinada como o produto da força média e da velocidade média. (5)

Velocidade média - A velocidade média foi obtida através da fórmula: velocidade final é igual à velocidade inicial, somada à aceleração multiplicada pelo tempo: $V_{f}=V_{i}+$ a.t. (5).

Plataforma de força - O Modelo da plataforma de Força usada foi da AMTI - Advanced Mechanical Technology, INC - modelo OR-6-5-2000 capacity. O limite máximo do modelo é de $10.000 \mathrm{~N}$ (aproximadamente $1000 \mathrm{Kg}$ ), podendo ser aplicado em qualquer lugar da superfície da plataforma ou $4.000 \mathrm{~N}$ (aproximadamente $400 \mathrm{Kg}$ ) de carga lateral aplicadas em qualquer lugar da plataforma nas direções $\mathrm{X}$ ou $\mathrm{Y}$.

Para o teste de força na plataforma algumas diretrizes são necessárias, o atleta foi instruído: comparecer para o teste de calção de banho (para possibilitar a colocação adequada dos marcadores) e para não participar no dia anterior de atividades físicas muito extenuantes. Os testes foram realizados sempre no período vespertino. Antes da medida de força de reação do solo se faz necessário a preparação do sujeito com a colocação dos marcadores que permitem a captação pelas câmeras de infravermelho.

Para que os movimentos sejam captados pelas câmeras de infravermelho, marcadores reflexivos foram colocados em pontos pré-determinados do corpo. As marcações protocoladas pelo sistema operacional Peak Motus foram modificas das marcações de Helen Hayes: 


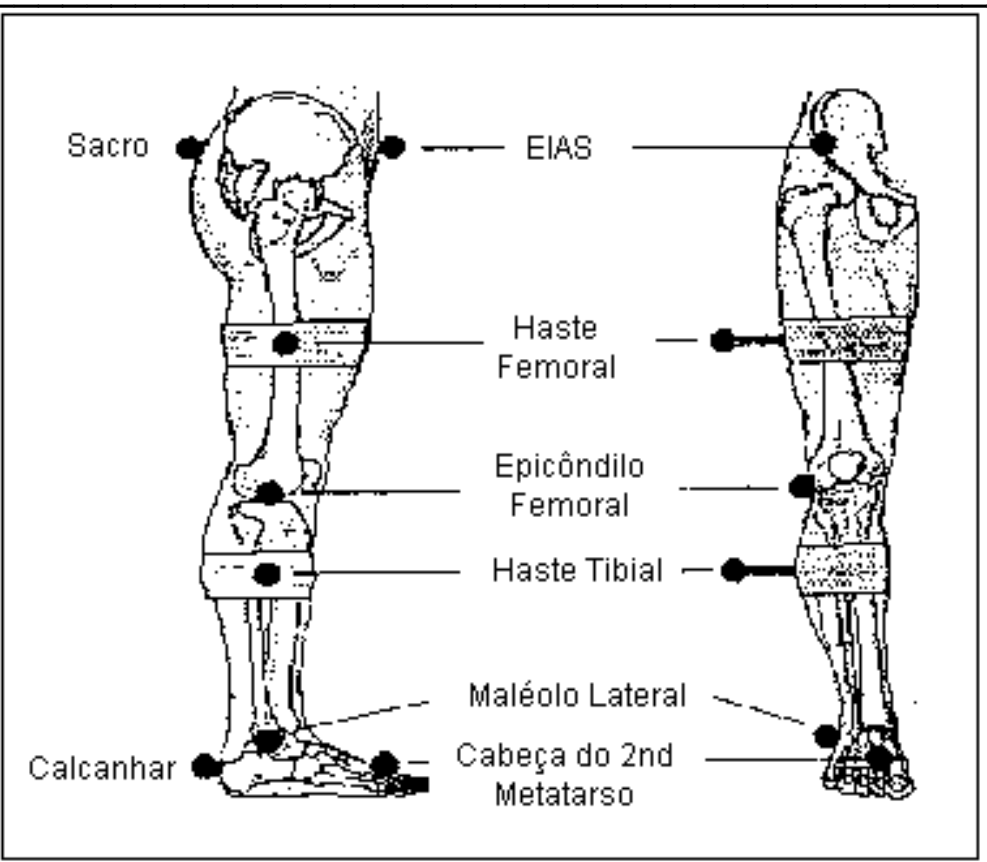

Figura 1- Ilustração dos pontos de colocação dos marcadores (figura adaptada do manual de instruções da plataforma de força AMTI - Modelo OR-6-5-2000 capacity).

Cada sujeito executou três saltos máximos, com o intervalo aproximado de quatro minutos, tais saltos foram registrados e todos foram selecionados para as análises propostas neste estudo.

\section{PROCEDIMENTOS DE ANÁLISE}

Análise estatística correlacional - Segundo Thomas e Nelson(23) a correlação é uma técnica estatística utilizada para determinar os relacionamentos entre duas ou mais variáveis. Foi utilizado à correlação produto momento de Pearson. Foram feitas análises estatísticas da correlação entre as seguintes variáveis: força máxima de reação do solo (força de saída e de chegada), força relativa, média da potência gerada, velocidade média e máxima impulsão vertical.

Análise dos resultados das variáveis- Após o estudo correlacional, apresentaremos as variáveis que mais influenciaram nos resultados da impulsão vertical e da potência média gerada.

Análise dos resultados individuais- Com os dados das variáveis dos testes foi elaborada uma tabela com os resultados dos saltos de cada sujeito em cada uma das três tentativas, e foi analisado as diferenças entre eles tomando como ponto de referência para a discussão o salto que atingiu uma maior altura vertical. 
Análise comparativa entre os saltos de maior e menor altura vertical- A partir de um ordenamento dos valores dos saltos verticais, partindo do maior para o menor, fizemos uma análise comparativa entre os dez saltos de maior altura e os dez de menor altura. Para isso foi elaborada uma tabela com as médias para cada variável estudada, para um cada dos grupos e a partir destas foi estabelecida a comparação.

\section{APRESENTAÇÃO DOS RESULTADOS}

Tabela 1- Distribuição da idade, peso, altura, massa corporal magra, percentual de gordura, média e desvio padrão de quinze sujeitos utilizados na pesquisa

\begin{tabular}{cccccc}
\hline Sujeito & Idade & Peso & Altura & MCM & \% gord. \\
\hline 1 & 21 & 73,9 & 173,5 & 59,8 & 19,0 \\
2 & 21 & 73,9 & 176,5 & 62,1 & 16,0 \\
3 & 25 & 71,7 & 175 & 58,5 & 18,5 \\
4 & 17 & 64,2 & 170,5 & 56,7 & 11,6 \\
5 & 23 & 76,1 & 178,5 & 63,5 & 16,5 \\
6 & 32 & 89,4 & 188,5 & 68,5 & 23,4 \\
7 & 30 & 72 & 170 & 56,3 & 21,7 \\
8 & 17 & 71,8 & 184 & 63,9 & 11,0 \\
9 & 33 & 82,5 & 172,5 & 61,9 & 24,9 \\
10 & 34 & 73,7 & 177 & 63,9 & 13,3 \\
11 & 34 & 69,2 & 177 & 60,4 & 12,7 \\
12 & 35 & 87,1 & 189 & 66,5 & 23,7 \\
13 & 24 & 79,2 & 183 & 68,6 & 13,4 \\
14 & 21 & 81 & 191,5 & 73,3 & 9,6 \\
15 & 21 & 81,9 & 191 & 71,2 & 13,1 \\
\hline Média & $\mathbf{2 5 , 8 7}$ & $\mathbf{7 6 , 5 1}$ & $\mathbf{1 7 9 , 8 3}$ & $\mathbf{6 3 , 7}$ & $\mathbf{1 6 , 6}$ \\
Des-Pad & $\mathbf{6 , 4 7}$ & $\mathbf{6 , 8 8}$ & $\mathbf{7 , 4 5}$ & $\mathbf{5 , 1}$ & $\mathbf{5 , 0}$ \\
\hline
\end{tabular}

Tabela 2- Distribuição da média geral de cada sujeito para as variáveis pesquisadas dos três saltos verticais realizados e a média geral e desvio padrão de todos os sujeitos. 


\begin{tabular}{|c|c|c|c|c|c|c|}
\hline Sujeitos & $\begin{array}{c}\text { Impul- } \\
\text { Vert. }\end{array}$ & Potência & $\begin{array}{c}\text { F- } \\
\text { Saída }\end{array}$ & $\begin{array}{c}\text { F- } \\
\text { Chegada }\end{array}$ & $\begin{array}{c}\text { F- } \\
\text { Relativa }\end{array}$ & Velocidade \\
\hline 1 & 0,273 & 1820,53 & 1572,76 & 2717,94 & 1,169 & 1,157 \\
\hline 2 & 0,352 & 2482,73 & 1889,69 & 2894,32 & 2,607 & 1,313 \\
\hline 3 & 0,297 & 1872,43 & 1550,25 & 2782,13 & 2,204 & 1,208 \\
\hline 4 & 0,277 & 1653,7 & 1416,3 & 2388,18 & 2,249 & 1,167 \\
\hline 5 & 0,290 & 1681,1 & 1412,94 & 2645,01 & 1,892 & 1,191 \\
\hline 6 & 0,290 & 2266,67 & 1900,86 & 2832,44 & 2,167 & 1,192 \\
\hline 7 & 0,214 & 1862,97 & 1820,35 & 2432,19 & 2,577 & 1,023 \\
\hline 8 & 0,285 & 1689,85 & 1430,15 & 2060,24 & 2,030 & 1,182 \\
\hline 9 & 0,223 & 1965,76 & 1880,54 & 2893,66 & 2,323 & 1,045 \\
\hline 10 & 0,253 & 1828,12 & 1643,52 & 2335,78 & 2,273 & 1,113 \\
\hline 11 & 0,358 & 2101,14 & 1585,78 & 1958,26 & 2,336 & 1,324 \\
\hline 12 & 0,316 & 2289,58 & 1838,73 & 2880,03 & 2,152 & 1,245 \\
\hline 13 & 0,316 & 2578,44 & 1937,3 & 2656,62 & 2,493 & 1,332 \\
\hline 14 & 0,252 & 1551,83 & 1400,56 & 2799,16 & 1,762 & 1,110 \\
\hline 15 & 0,223 & 1534,62 & 1464,68 & 2747,25 & 1,823 & 1,047 \\
\hline Média & 0,281 & 1945,30 & 1649,63 & 2601,55 & 2,137 & 1,176 \\
\hline Desvpad & 0,044 & 329,63 & 206,42 & 300,59 & 0,368 & 0,099 \\
\hline
\end{tabular}

Tabela 3- Resultados da correlação (r) entre as variáveis estudadas.

\begin{tabular}{|c|c|c|c|c|c|c|c|}
\hline & Salto Vert. & Potência & F-Saída & F-Cheg. & F-Rel. & Veloc. & Peso \\
\hline Salto Vert. & & & & & & & \\
\hline Potência & 0,701 & & & & & & \\
\hline F- Saída & 0,235 & 0,856 & & & & & \\
\hline F- Cheg. & $-0,142$ & 0,012 & 0,10 & .............. & ..... & & \\
\hline F-Rel. & 0,319 & 0,699 & 0,74 & $-0,04$ & ……?. & & \\
\hline Veloc. & 0,999 & 0,688 & 0,22 & $-0,14$ & 0,30 & & \\
\hline Peso & $-0,102$ & 0,286 & 0,44 & 0,21 & $-0,27$ & $-0,10$ & \\
\hline $\mathrm{MCM}$ & 0,01 & 0,033 & 0 & 0,15 & $-0,54$ & 0,00 & 0,729 \\
\hline
\end{tabular}

COMENTÁRIOS SOBRE OS RESULTADOS DE CORRELAÇÃO APONTADOS NA TABELA 03 
$\overline{\text { A correlação entre salto vertical e potência foi significativa }(\mathrm{r}=0,701 ; \mathrm{P}<0,001) \text {; a correlação entre salto }}$ vertical e força de saída não foi significativa; a correlação entre salto vertical e força relativa foi significativa $(\mathrm{r}=0,319 ; \mathrm{P}<0,05)$; a correlação entre salto vertical e velocidade foi extremamente significativa $(\mathrm{r}=0,999 ; \mathrm{P}<0,001)$; a correlação entre salto vertical e peso corporal foi negativa e não significativa e a correlação entre salto vertical e massa corporal magra não foi significativa.

A correlação entre potência e força de saída foi significativa $(\mathrm{r}=0,856 ; \mathrm{P}<0,001)$; a correlação entre a potência e a força de chegada não foi significativa; a correlação entre potência e força relativa foi significativa $(r=0,699 ; P<0,001)$; a correlação entre potência e velocidade foi significativa $(r=0,688$; $\mathrm{P}<0,001)$; a relação entre potência e peso foi significativa $(\mathrm{r}=0,286 ; \mathrm{P}<0,10)$ e a relação entre potência e massa corporal magra não foi significativa.

A correlação entre força de saída e força de chegada não foi significativa; a correlação entre força de saída e força relativa foi significativa $(\mathrm{r}=0,74 ; \mathrm{P}<0,001)$; a correlação entre força de saída e velocidade não foi significativa; a correlação entre força de saída e peso corporal foi significativa $(r=0,44 ; \mathrm{P}<0,01)$ e a correlação entre força de saída e massa corporal magra não foi significativa.

A força de chegada não mostrou correlação significativa com nenhuma das outras variáveis.

A correlação entre a força relativa e a velocidade foi significativa $(r=0,30 ; P<0,10)$; a correlação entre força relativa e peso corporal foi significativa $(r=-0,27 ; \mathrm{P}<0,10)$ e a correlação entre a força relativa e a massa corporal magra foi ainda mais significativa $(r=-0,54 ; \mathrm{P}<0,001)$.

A velocidade não mostrou correlação significativa nem com o peso e nem com a massa corporal magra.

$\mathrm{O}$ peso corporal e a massa corporal magra apresentaram uma correlação significativa $(\mathrm{r}=0,729$; $\mathrm{P}<0,001)$.

\section{APRESENTAÇÃO DOS VALORES DOS DEZ SALTOS QUE ATINGIRAM MAIOR ALTURA E DOS DEZ QUE ATINGIRAM MENOR ALTURA}

Os quarenta e cinco resultados dos saltos (três para cada sujeito) foram classificados do maior para o menor e do menor para o maior e desta classificação foram retiradas às tabelas a seguir.

Conexões: revista da Faculdade de Educação Física da UNICAMP, Campinas, v. 8, n. 1, p. 38-53, jan./abr. 2010. 
Tabela 4- Resultados dos dez saltos de maior altura vertical extraídos da tabela 01

\begin{tabular}{|c|c|c|c|c|c|c|}
\hline Salto-Suj & Impulsão & Potência & F-Saída & F-chegada & F-Relativa & Velocidade \\
\hline Salto $01(13)$ & 0,375 & 2667,959 & 1966,473 & 2829,145 & 2,531 & 1,357 \\
\hline Salto 01(11) & 0,371 & 2174,733 & 1611,964 & 1895,186 & 2,375 & 1,349 \\
\hline Salto 02(13) & 0,371 & 2502,015 & 1854,553 & 2469,631 & 2,387 & 1,349 \\
\hline Salto $02(02)$ & 0,368 & 2627,522 & 1956,473 & 2875,49 & 2,699 & 1,343 \\
\hline Salto 02(11) & 0,364 & 2116,059 & 1583,441 & 2033,35 & 2,333 & 1,336 \\
\hline Salto $03(02)$ & 0,346 & 2472,068 & 1897,193 & 2894,635 & 2,617 & 1,303 \\
\hline Salto $01(02)$ & 0,341 & 2348,607 & 1815,427 & 2912,861 & 2,504 & 1,294 \\
\hline Salto 03(11) & 0,339 & 2012,637 & 1561,947 & 1946,244 & 2,301 & 1,289 \\
\hline Salto $03(13)$ & 0,339 & 2565,335 & 1990,88 & 2671,084 & 2,562 & 1,289 \\
\hline Salto $02(12)$ & 0,328 & 2313,814 & 1823,799 & 2851,069 & 2,134 & 1,269 \\
\hline
\end{tabular}

Tabela 5. Resultados dos dez saltos de menor altura vertical extraídos da tabela 01

\begin{tabular}{|c|c|c|c|c|c|c|}
\hline Salto-Suj & Impulsão & Potência & F-Saída & F-chegada & F-Relativa & Velocidade \\
\hline Salto 01(07) & 0,2326 & 1969,402 & 1843,899 & 2375,583 & 2,321 & 1,068 \\
\hline Salto $02(07)$ & 0,2300 & 1892,325 & 1781,552 & 2592,369 & 2,217 & 1,062 \\
\hline Salto $01(15)$ & 0,227 & 1475,666 & 1398,974 & 2880,407 & 1,741 & 1,055 \\
\hline Salto 03(09) & 0,226 & 1898,353 & 1804,308 & 2912,306 & 2,555 & 1,052 \\
\hline Salto 01(09) & 0,225 & 2118,103 & 2015,521 & 2862,195 & 2,490 & 1,051 \\
\hline Salto 02(09) & 0,224 & 1910,074 & 1821,818 & 2906,5 & 2,251 & 1,048 \\
\hline Salto $03(07)$ & 0,2189 & 1902,021 & 1835,606 & 2328,632 & 2,268 & 1,036 \\
\hline Salto $02(15)$ & 0,214 & 1470,003 & 1434,976 & 2502,003 & 1,786 & 1,024 \\
\hline Salto $03(15)$ & 0,210 & 1581,731 & 1560,103 & 2859,354 & 2,209 & 1,014 \\
\hline Salto $02(14)$ & 0,206 & 1429,810 & 1424,038 & 2903,145 & 2,016 & 1,004 \\
\hline
\end{tabular}


Tabela 6-Tabela comparativa entre a média e o desvio padrão das variáveis estudadas para os dez maiores e os dez menores resultados de impulsão vertical respectivamente.

\begin{tabular}{cccccccc}
\hline $\begin{array}{c}\text { Salto } \\
\text { Suj. }\end{array}$ & Impulsão & Potência & $\begin{array}{c}\text { Força } \\
\text { Saída }\end{array}$ & $\begin{array}{c}\text { Força } \\
\text { Chegada }\end{array}$ & $\begin{array}{c}\text { Força } \\
\text { Relativa }\end{array}$ & $\begin{array}{c}\text { Velocidade } \\
\text { Gordura }\end{array}$ \\
\hline Média & 0,354 & 2380,075 & 1806,215 & 2537,870 & 2,444 & 1,318 & 16,450 \\
\hline D-Padrão & 0,017 & 224,604 & 163,435 & 422,273 & 0,169 & 0,032 & 4,360 \\
\hline Média & 0,221 & 1761,103 & 1692,080 & 2712,249 & 2,196 & 1,042 & 18,200 \\
\hline D-Padrão & 0,009 & 220,024 & 217,811 & 237,037 & 0,355 & 0,021 & 5,370 \\
\hline
\end{tabular}

Os valores médios da altura vertical no salto mostram uma diferença de 13,3 centímetros, com o grupo de menor resultado atingindo apenas $62,4 \%$ da altura conseguida pelos melhores saltadores.

Os valores médios da potência mostraram uma diferença de 618,972 Watts, com o grupo de menor resultado atingindo aproximadamente $74 \%$ da potência apresentada pelos melhores saltadores.

Os valores médios da força de saída mostraram uma diferença de 103,404 Newtons, com o grupo de menor resultado atingindo aproximadamente 93,68\% da força de saída apresentada pelos melhores saltadores.

Os valores médios da força de chegada mostraram uma diferença de 174,379 Newtons, o grupo de menor resultado nos saltos verticais apresentou uma média maior para os valores desta variável, aproximadamente $6,5 \%$ maior que os grupos dos melhores saltadores.

Os valores médios da força de relativa mostraram uma diferença 0,248 , com o grupo de menor valor nos saltos atingindo aproximadamente $89,8 \%$ dos valores apresentados pelo grupo dos melhores saltadores.

Os valores médios da velocidade mostram uma diferença de $0,276 \mathrm{~m} / \mathrm{s}^{2}$, com o grupo de menor valor nos saltos atingindo aproximadamente $75 \%$ da velocidade apresentada pelo grupo dos melhores saltadores.

Para os valores de percentual de gordura o grupo dos saltadores de menor altura nos saltos apresentou um percentual mais elevado, cerca de $1,75 \%$ de diferença em relação ao outro grupo, em média 9,6\% a mais do que o grupo de maior resultado nos saltos. Entre os quatro sujeitos que fizeram os dez maiores resultados nos saltos, três deles estavam entre $12 \%$ e $16 \%$ de percentual de gordura, apenas um deles, o que conseguiu apenas o décimo valor no salto, está acima disto. Isto nos faz deduzir que estar dentro de padrões aceitáveis de percentual de gordura pode ser fator positivo para a capacidade de salto. 


\section{DISCUSSÃO}

A correlação entre as variáveis pesquisada para o salto vertical nos mostrou que a variável de maior influência foi a velocidade média, o que é possível de ser explicado pela relação intima entre os dois elementos expressa pela física $(V=D / t)$, mas os valores encontrados foram mais altos que os esperados, um $r=0,999 ; P<0,001$.

A potência foi o segundo item que mostrou uma maior correlação com a altura atingida no salto vertical, um $\mathrm{r}=0,701 ; \mathrm{P}<0,001$ é bastante significativo. Se analisarmos a partir do coeficiente de correlação $\left(\mathrm{r}^{2}\right)$ verificaremos que $49 \%$ da variação na altura do salto pode ser explicada pela potência gerada.

Um outro fator que mostrou correlação significativa com a altura do salto vertical foi a força relativa $(\mathrm{r}=$ $0,319 ; \mathrm{P}<0,05)$ que nos confirma, que a relação entre a força aplicada para o salto e o peso corporal é fator interveniente da qualidade do mesmo, com um $\mathrm{r}^{2}=10,19 \%$. Sendo a força relativa a razão entre a máxima força de reação do solo e o peso corporal, podemos observar que o coeficiente de determinação para a força de saída em relação ao salto vertical $r^{2}=5,526 \%$ é menor do que o coeficiente de determinação $\left(\mathrm{r}^{2}\right)$ para a força relativa. Isso nos confirma as propostas apresentadas pela literatura de que quanto maior a força menor a velocidade e conseqüentemente menor a altura do salto vertical.

Com relação a potência, a variável que foi melhor correlacionada com esta foi a força de saída $(\mathrm{r}=$ $0,856 ; \mathrm{P}<0,001)$. A relação entre as variáveis apresenta $r^{2}=73,38 \%$, o que nos mostra que a melhor capacidade de produção de potência depende em grande parte da força de saída. A necessidade da melhoria da força de membros inferiores para a incrementação dos resultados da potência é evidente, reforçado ainda pela significativa relação entre a potência e a força relativa $(r=0,699 ; \mathrm{P}<0,001)$ que apresentou um $r^{2}=48,8 \%$. A velocidade apresentou uma correlação também significativa ( $r=$ $0,688 ; \mathrm{P}<0,001)$ com $\mathrm{r} 2=47,28 \%$. As variáveis estudadas apresentaram um efeito em "sequência", em "cascata" até chegar ao salto vertical. A força relativa apresentou uma grande correlação com a força de saída do solo, a força de saída apresentou uma grande correlação com a potência e a potência uma grande correlação com a máxima altura atingida no salto vertical. É interessante observar que a velocidade mostrou correlação significativa com três das seis vaiáveis pesquisadas: velocidade $\mathrm{x}$ salto vertical $(\mathrm{r}=$ 0,999; $\mathrm{P}<0,001$ e $\left.\mathrm{r}^{2}=99 \%\right)$, velocidade $\mathrm{x}$ potência $\left(\mathrm{r}=0,688 ; \mathrm{P}<0,001\right.$ e $\left.\mathrm{r}^{2}=47,28 \%\right)$, velocidade $\mathrm{x}$ força relativa $(\mathrm{r}=0,30 ; \mathrm{P}<0,10$ e $\mathrm{r} 2=9,01 \%)$. 
$\overline{\mathrm{A} \text { força de chegada não mostrou correlação significativa com nenhuma das variáveis estudadas, mas nos }}$ mostrou o efeito do treinamento com saltos pliométricos. A força de chegada no solo foi sempre maior do que a de saída para todos os sujeitos do estudo.

O sujeito que obteve a primeira, a terceira e a nona maior altura no salto, produziu também os melhores valores para: potência, velocidade e força de chegada. O sujeito que obteve a segunda, a quinta e a oitava maior altura no salto, produziu também os melhores valores para: potência, velocidade, força de saída e força relativa. O sujeito que obteve a quarta, a sexta e a sétima maior altura no salto, produziu também os melhores valores para: potência, velocidade, força de saída e força relativa. O sujeito que obteve a décima maior altura no salto teve uma outra única variável como de melhor resultado, a velocidade.

A massa corporal magra na correlação com a força relativa $\left(r=-0,54 ; \mathrm{P}<0,001\right.$ e $\left.\mathrm{r}^{2}=29 \%\right)$ mostrou ser mais significativa do que o peso corporal $\left(\mathrm{r}=-0,27 ; \mathrm{P}<0,10\right.$ e $\left.\mathrm{r}^{2}=7,48 \%\right)$. Isso nos faz deduzir que possuir um percentual de gordura menor não faz diferença no salto automaticamente, se faz necessário que a massa corporal magra restante excetuada o percentual de gordura possua uma musculatura o mais forte possível para ser capaz de gerar uma boa força de saída do solo.

É interessante apontar que a correlação entre a força relativa e a velocidade foi significativa $(r=0,30$; $\mathrm{P}<0,10)$; a correlação entre força relativa e peso corporal foi significativa $(\mathrm{r}=-0,27 ; \mathrm{P}<0,10)$ e a correlação entre a força relativa e a massa corporal magra foi ainda mais significativa.

\section{CONCLUSÕES}

$1^{\text {a }} \mathrm{A}$ análise correlacional, a análise individual e a análise comparativa confirmaram como fator de maior importância para se obter uma boa capacidade de salto as variáveis: velocidade e potência.

$2^{\mathrm{a}}$ A potência é muito influenciada pela força de saída do solo, o que nos remete à necessidade de desenvolver uma força significativa de membros inferiores e a referência para isso foi dada pela força relativa.

$3^{\text {a }}$ Os sujeitos que conseguiram maior resultado no salto apresentaram uma maior performance também em pelo menos três outras variáveis, o que nos faz deduzir que a ação conjunta destas variáveis é fator determinante de boa performance.

$4^{\mathrm{a}} \mathrm{A}$ força de chegada não apresentou correlação significativa com nenhuma das variáveis estudadas. 
$5^{\text {a }}$ O sujeito executa uma força maior no amortecimento da queda trabalhando no ciclo excêntrico-concêntrico, o que é um dos fatores importantes no desenvolvimento de trabalhos pliométricos.

$6^{\text {a }}$ Os resultados apresentados pelo estudo podem servir de bases para novas pesquisas que de fato se fazem necessárias, mas já fornece importantes referências para atletas e treinadores reverem as bases de força geral e específica dos seus treinamentos o salto vertical.

\section{REFERÊNCIAS}

DURARD, B.R. Movimento funcional humano: mensuração e análise. São Paulo, Manole, 2001.

ZAKHAROV, A. Ciência do treinamento desportivo. Traduzido por: Antonio Carlos Gomes. Rio de Janeiro: Grupo Palestra Sport, 1992.

WEINECK, J. Manual de treinamento esportivo. Traduzido por: Maria Ermatina Galvão Gomes Pereira et al. São Paulo: Manole, 1989.

VERKHOSHANSKI, I. Treinamento Desportivo: teoria e metodologia. Porto Alegre: Artmed, 2001.

ENOKA, R.M. Bases neuromecânicas da cinesiologia. São Paulo: Manole, 2000.

KELLER, F.J.; GETTYS, W.E.; SKOVE, M.J. Física. Traduzido por: Alfredo Alves de Farias. São Paulo: Makron Books, v. 1, 1997.

BOMPA T.O. Periodização: teoria e metodologia do treinamento. São Paulo: Phorte, 2002.

MOREIRA, S. B. Determinação da Força Relativa a Partir de Testes Práticos de Resistência Muscular Localizada. Artus. v. 22. p. 58-62, 1989.

ZATSIORSKY, W.M. Ciência e prática do treinamento de força. São Paulo: Phorte, 1999.

BOSCO, C.; KOMI, P.V. Influence of aging on the behavior of leg extensor muscles. Eur. J of App Physiology, v. 45, p. 209- 19, 1980.

BOSCO. C.; TIHANYI, J.; KOMI, P.V.; FEKETE, G.; APOR, P. Store and recoil of elastic energy in slow and fast types of human skeletal muscles. Act Phy Scand, 116:343-9, 1982.

HUDSON, J.L. Cordination of segments in the vertical jump. Med and Sci in Spo and Exercise, v 18, $\mathrm{n}$. 2, p. 242-251, 1986.

BOBBERT, M.F. Effects of muscle strengthening on vertical jump height: a simulation study. Med and Sci in Sport and Exercise, v. 26, n.8, p.1012-20, 1987. 
PEREIRA, L.F.R. Desenvolvimento de um sistema computadorizado para estudo de saltos verticais consecutivos. Rio de Janeiro: Dissertação (Mestrado) - Escola de Educação Física e Desportos, Centro de Ciências da Saúde, UFRJ, 1987.

HARMAN, E.A.; et al, The effects of arms and countermovement on vertical jumping. Med and Sci in Spo and Exercise, v.22, n.6., p. 825-33, 1990.

DAVIES, B.N.; JONES, K.G. An analysis of the performance of male students in the vertical and standing long jump tests and the contribution of arm swinging. J of Hum Mov Studies, v. 24, p. 25-38, 1993.

HOLCOMB, W.R.; LANDER. J.E.; RUTLAND, R.M. A biomechanical analysis of the vertical jump and three modified plyometric depth jumps. J Stre an Cond Research. v. 10, n. 2, p. 83-88, 1996.

CORDOVA, M.L.; ARMISTRONG, C.W. Reliability of ground reaction forces during a vertical jump: implications for functional strength assessment. J of Ath Training, v. 31, n. 4, p. 342-345, 1996.

LANDESBERG, A.; SIDEMAN, S. Force-velocity relationship and biochemical-to-mechanical energy conversion by the sarcomere. Heart and Circ Physiology. v. 278, p. 1274-1284, 2000.

GALDI, E.H.G. Performance da resistencia muscular de membros inferiores em praticantes da modalidade esportiva voleibol, através do salto vertical. 125p. Tese (Doutorado)- Universidade Estadual de Campinas, Campinas, 1999.

KUBO, K.; KAWAKAMI, Y.; FUKUNAGA, T. Influence of elastic properties of tendon strutures on jump performance in humans. Journal of Applied Physiology. v. 87, p. 2090-96, 1999.

KUROKAWA, S.; FUKUNAGA, T.; FUKASHIRO, S. Behavior of fascicles na tendinous strutures of human gastrocnemius during vertical jumping. J of App Physiology. v. 90, p. 1349-58, 2001.

THOMAS, J.R.; NELSON, J.K. Métodos de pesquisa em atividade fisica. 3 ed. Porto Alegre: Artmed, 2002. 
Emerson Miguel da Cruz

Faculdade de Educação Física - ESEFEGO

Antonia Dalla Pria Bankoff

Laboratório de Avaliação Postural - Faculdade de Educação Física

Universidade Estadual de Campinas

Av. Érico Veríssimo, 701 - CP 6134

Cidade Universitária - Campinas - SP

CEP: 13083-851

E-mail bankoff@fef.unicamp.br

Referencia do artigo:

ABNT

CRUZ, E. M.; BANKOFF A. D. P. Estudo do Salto Vertical Máximo: Análise da Correlação de Forças Aplicadas. Conexões, v. 8, n. 1, p. 38-53, 2010

APA

Cruz, E. M., Bankoff A. D. P. (2010). Estudo do Salto Vertical Máximo: Análise da Correlação de Forças Aplicadas. Conexões, 8(1), 38-53

\section{VANCOUVER}

Cruz EM, Bankoff ADP. Estudo do Salto Vertical Máximo: Análise da Correlação de Forças Aplicadas. Conexões, 2010 8(1): 38-53

Recebido em 20/12/2009

Aceito para Publicação em fev. 2010 